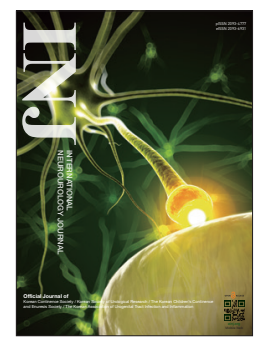

\title{
Afferent Pathway-Mediated Effect of a1 Adrenergic Antagonist, Tamsulosin, on the Neurogenic Bladder After Spinal Cord Injury
}

\author{
Jin-Hee Han ${ }^{1}$, Sung-Eun Kim², Il-Gyu Ko², Jayoung Kim ${ }^{3,4}$, Khae Hawn Kim ${ }^{5}$ \\ ${ }^{1}$ Department of Anesthesiology and Pain Medicine, Kyung Hee Medical Center, College of Medicine, Kyung Hee University, Seoul, Korea \\ ${ }^{2}$ Department of Physiology, College of Medicine, Kyung Hee University, Seoul, Korea \\ ${ }^{3}$ Department of Surgery and Biomedical Sciences, Cedars-Sinai Medical Center, Los Angeles, CA, USA \\ ${ }^{4}$ Department of Medicine, University of California Los Angeles, Los Angeles, CA, USA \\ ${ }^{5}$ Department of Urology, Gachon University Gil Medical Center, Gachon University School of Medicine, Incheon, Korea
}

Purpose: The functions of the lower urinary tract (LUT), such as voiding and storing urine, are dependent on complex central neural networks located in the brain, spinal cord, and peripheral ganglia. Thus, the functions of the LUT are susceptible to various neurologic disorders including spinal cord injury (SCI). SCI at the cervical or thoracic levels disrupts voluntary control of voiding and the normal reflex pathways coordinating bladder and sphincter functions. In this context, it is noteworthy that al-adrenoceptor blockers have been reported to relieve voiding symptoms and storage symptoms in elderly men with benign prostatic hyperplasia (BPH). Tamsulosin, an al-adrenoceptor blocker, is also considered the most effective regimen for patients with LUT symptoms such as BPH and overactive bladder (OAB).

Methods: In the present study, the effects of tamsulosin on the expression of c-Fos, nerve growth factor (NGF), and nicotinamide adenine dinucleotide phosphate-diaphorase (NADPH-d) in the afferent micturition areas, including the pontine micturition center (PMC), the ventrolateral periaqueductal gray matter (vlPAG), and the spinal cord (L5), of rats with an SCI were investigated.

Results: SCI was found to remarkably upregulate the expression of c-Fos, NGF, and NADPH-d in the afferent pathway of micturition, the dorsal horn of L5, the vlPAG, and the PMC, resulting in the symptoms of OAB. In contrast, tamsulosin treatment significantly suppressed these neural activities and the production of nitric oxide in the afferent pathways of micturition, and consequently, attenuated the symptoms of $\mathrm{OAB}$.

Conclusions: Based on these results, tamsulosin, an a1-adrenoceptor antagonist, could be used to attenuate bladder dysfunction following SCI. However, further studies are needed to elucidate the exact mechanism and effects of tamsulosin on the afferent pathways of micturition.

Keywords: Spinal cord injury; Tamsulosin; c-Fos; Nerve growth factor; Nitric oxide synthase

- Grant/Fund Support: This study was supported by a grant from the National Research Foundation of Korea (NRF-2017R1A2B2005412).

- Research Ethics: We followed the guidelines of the Institutional Care and Use Committee of Kyung Hee University during all laboratory procedures (KHUASP[SE]-17-093), and all experiments were performed in accordance with the guiding principles for the care and use of animals approved by the Council of the National Institutes of Health Guide for the Care and Use of Laboratory Animals.

- Conflict of Interest: KHK, an associate editor of INJ, is the corresponding author of this article. However, he played no role whatsoever in the editorial evaluation of this article or the decision to publish it. Except for that, no potential conflict of interest relevant to this article was reported.

\section{- HIGHLIGHTS}

- Tamsulosin, an $\mathrm{a}_{1}$-adrenoceptor antagonist, showed beneficial effects on alleviating the symptoms associated with bladder hyperactivity by suppressing hyperexcitability in the afferent pathways of micturition in spinal cord injury.

Corresponding author: Khae Hawn Kim (iD https://orcid.org/0000-0002-7045-8004 Department of Urology, Gachon University School of Medicine, Gil Medical Center, 21 Namdong-daero 774 beon-gil, Namdong-gu, Incheon 21565, Korea Tel: +82-32-460-3334, Fax: +82-32-460-8414, E-mail: kimcho99@gilhospital.com Submitted: September 11, 2017 / Accepted after revision: September 22, 2017
(7) $\$$ This is an Open Access article distributed under the terms of the Creative Commons Attribution Non-Commercial License (http://creativecommons.org/licenses/by-nc/4.0/) which permits unrestricted non-commercial use, distribution, and reproduction in any medium, provided the original work is properly cited. 


\section{ITRODUCTION}

The normal function of the lower urinary tract (LUT) requires coordination of the urinary bladder, urethra, pelvic floor, efferent and afferent neurons, and specific spinal cord and brain areas [1]. The bladder and external urethral sphincter are innervated directly or indirectly by many central nervous system (CNS) regions, such as the pontine micturition center (PMC), locus coeruleus, hypothalamus, and preoptic area [2]. The PMC, as the supraspinal switching center, is known to regulate the storage and elimination of urine [3]. The periaqueductal gray matter (PAG) of the hypothalamus has also been suggested to be a critical component of the micturition reflex, and interneurons in the lumbosacral cord strongly project to the lateral and dorsal parts of the PAG [4].

The central micturition regions, including the PAG and PMC, are activated by stimulation of the bladder. Many studies have reported that bladder stimulation caused the expression of c-Fos in the PAG and PMC related to micturition $[3,5]$. The transcription factor c-Fos, an immediate early gene, has been used as a marker of neuronal activity [6]. Nerve growth factor (NGF), the prototypical neurotrophic factor, has also been reported to modulate neuronal function along micturition pathways, and to serve as a urinary marker of bladder overactivity [7].

Nitric oxide (NO) is considered to be an important neurotransmitter and cell signaling molecule with a broad range of functions in the LUT. NO has been reported to play an important role in the micturition process and in disorders of the LUT [8]. NO is synthesized from L-arginine by nitric oxide synthase (NOS), which is classified into 3 groups: neuronal NOS (nNOS), inducible NOS, and endothelial NOS. Zhang et al. [9] reported that NO participates in the neural pathways controlling the LUT, and that NOS alterations may be involved in LUT dysfunction.

As described above, the functions of the LUT, such as voiding and storing urine, are dependent on neural circuits located in the brain, spinal cord, and peripheral ganglia. Therefore, the functions of the LUT are susceptible to various neurologic disorders including spinal cord injury (SCI) [10]. SCI may result from traumatic insult, such as spinal transection or contusion, or ischemic insult occurring at different levels of the spinal cord [11], and these injuries generate a range of events compromising the integrity of the neuronal pathways running through the spinal cord [12]. Virtually, SCI at the cervical or thoracic levels disrupts voluntary control of voiding and the normal reflex pathways that coordinate bladder and sphincter functions [13]. Thus, SCI animal models have been used to study the mechanism of detrusor overactivity and detrusor-sphincter dyssynergia [14].

The most commonly used first-line drugs for male patients with lower urinary symptoms are $\alpha 1$-adrenoceptor blockers, such as silodosin and prazosin, which relieve voiding symptoms and storage symptoms in elderly men with BPH by exerting an inhibitory effect on afferent input from the LUT [15].

Tamsulosin, an al-adrenoceptor blocker, is also considered the most effective regimen for patients with LUT symptoms suggestive of benign prostatic hyperplasia, and has been reported to play a role in the management of overactive bladder $(\mathrm{OAB})$ in women [16]. In addition, tamsulosin has been reported to improve storage function by inhibiting C-fiber mediated urethral afferent neurons [17]. Abrams et al. [18] studied the efficacy and safety of tamsulosin in patients with suprasacral spinal cord lesions, and suggested that long-term tamsulosin treatment improves bladder storage and emptying, alleviating the symptoms of autonomic dysreflexia. However, they did not propose an exact mechanism of the effects of tamsulosin on the afferent micturition regions. Therefore, the effects of tamsulosin on the expression of c-Fos, NGF and nicotinamide adenine dinucleotide phosphate-diaphorase (NADPH-d) in the afferent micturition areas, such as the PMC, the ventrolateral PAG (vlPAG), and the spinal cord (L5), of rats with an SCI were investigated in this study.

\section{MATERIALS AND METHODS}

\section{Animals}

Female Sprague-Dawley rats weighing $260 \pm 10 \mathrm{~g}$ (13 weeks of age) were used in this study. All experimental procedures were carried out in accordance with the Guidelines for the Care and Use of Animals approved by the Council of the National Institutes of Health Guide for the Care and Use of Laboratory Animals. The study was approved by the Institutional Care and Use Committee of Kyung Hee University (KHUASP[SE]-17-093). To minimize inadvertent c-Fos protein expression induced by environmental stimuli, the animals were gently and carefully handled during the experimental process. The animals were housed under controlled temperature $\left(22^{\circ} \mathrm{C} \pm 2^{\circ} \mathrm{C}\right)$ and lighting (8 AM to $8 \mathrm{PM}$ ) conditions and were supplied with food and water ad libitum. 
The animals were randomly divided into the following 4 groups ( $\mathrm{n}=8$ in each group): the sham group, the SCI-induced group, the SCI-induced and $0.01 \mathrm{mg} / \mathrm{kg}$ tamsulosin-treated group, and the SCI-induced and $1 \mathrm{mg} / \mathrm{kg}$ tamsulosin-treated group. Starting one day after the induction of SCI, the tamsulosin-treated groups received tamsulosin (Harunal, Astellas Pharma Inc., Tokyo, Japan) orally once a day for 3 weeks.

\section{Surgical Procedure for Inducing SCI}

The SCI model was induced as previously described [19]. The rats were anesthetized by inhalation of isoflurane ( $2 \%$ isoflurane in $30 \% \mathrm{O}_{2}$ and $70 \% \mathrm{~N}_{2}$, JW pharmaceutical, Seoul, Korea) during surgery. The skin in the T10-T12 areas was incised through a $2.5-\mathrm{cm}$ median incision, and the thoracic vertebral column and spinous process in the T11 were exposed by dissection. After dissection, the spinous process in T11 was ground using a drill until the vertebral arch area was reached, and a hole $2 \mathrm{~mm}$ deep from the surface of the vertebral arch was made using a surgical drill (diameter, $1 \mathrm{~mm}$ ), the spinal cord was damaged by a 22-G needle, and the hole was sealed (Fig. 1). The skin was closed layer by layer with continuous 3-0 silk sutures. The body temperature was maintained at $36^{\circ} \mathrm{C}$ $\pm 0.5^{\circ} \mathrm{C}$ during the induction of SCI using a Homeothermic Blanket Control Unit (Harvard Apparatus, Holliston, MA, USA) that enveloped the body and the head. After recovery, the animals were monitored for an additional 2 hours to prevent hypothermia. The animals in the sham-operation group were treated identically, except that the spinal cords was not damaged after the skin incisions.

\section{Cystometry}

Bladder function was evaluated using cystometry, as previously described [20]. The rats were tested via cystometry after 3 weeks of tamsulosin treatment. The rats were anesthetized with Zoletil 50 (10 mg/kg, intraperitoneally; Vibac Laboratories, Carros, France). A sterile polyethylene catheter (PE50) was inserted into the urethra through the bladder dome. The catheter was connected to a pressure transducer (Harvard Apparatus) and syringe pump (Harvard Apparatus) via a 3-way stopcock to record the intravesical pressure and to infuse saline into the bladder. After the bladder was emptied, cystometry was performed with saline infused at $0.5 \mathrm{~mL}$. The basal contraction pressure and contraction time in the bladder were monitored using Labscribe software (iWorx/CB Science Inc., Dover, DE, USA).

\section{Tissue Preparation}

The animals were sacrificed immediately after determining their voiding function by cystometry as previously described $[19,20]$. For the histological examination, the rats were transcardially perfused with 50mM phosphate-buffered saline (PBS), followed by $4 \%$ paraformaldehyde in $100 \mathrm{mM}$ sodium phos-
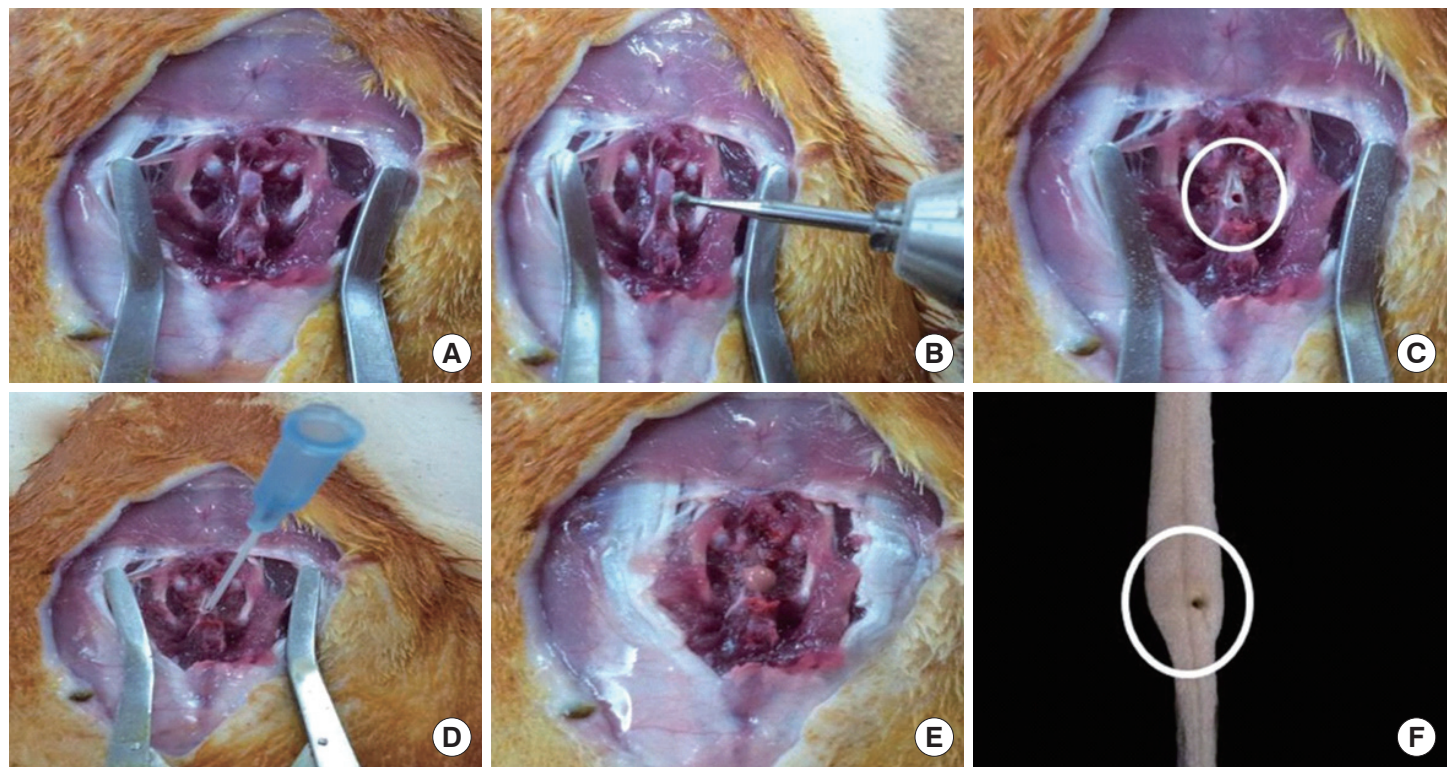

Fig. 1. Process of spinal cord injury induction. (A) Exposure of the spinous process. (B) Grinding the spinous process. (C) Hole in the vertebral arch. (D) Damage made with a needle in the spinal cord. (E) Sealing the hole. (F) The damaged region. 
phate buffer at $\mathrm{pH}$ 7.4. The brain was removed, postfixed in the same fixative overnight, and transferred into a $30 \%$ sucrose solution for cryoprotection. Serial $40-\mu \mathrm{m}$-thick coronal sections were made with a freezing microtome (Leica, Wetzlar, Germany). The PMC was selected from the region spanning from Bregma -9.68 to $-9.80 \mathrm{~mm}$. The vlPAG was selected from the region spanning from Bregma -7.64 to $-8.00 \mathrm{~mm}$, and the spinal cord was selected from the region spanning L5. Four sections on average in each region were collected from each rat.

\section{Immunohistochemistry for c-Fos and NGF}

To detect c-Fos expressions in the vlPAG, PMC, and spinal cord (L5) regions, c-Fos and NGF immunohistochemistry was performed as previously described [20]. Briefly, tissue sections were incubated overnight with rabbit anti-c-Fos and mouse anti-NGF-antibodies (Santa Cruz Biotechnology Inc., Santa Cruz, CA, USA) at a dilution of 1:1,000. The sections were then incubated for 1 hour with anti-rabbit and anti-mouse secondary antibodies (1:200; Vector Laboratories, Burlingame, CA, USA). The sections were subsequently incubated with an avidin-biotin-peroxidase complex (1:100; Vector Laboratories) for 1 hour at room temperature. Immunoreactivity was visualized by incubating the sections in a solution consisting of $0.02 \% 3,3^{\prime}$-diaminobenzidine tetrahydrochloride (Sigma-Aldrich, St. Louis, $\mathrm{MO}, \mathrm{USA}$ ) and $0.03 \% \mathrm{H}_{2} \mathrm{O}_{2}$ in $50 \mathrm{mM}$ Tris- $\mathrm{HCl}(\mathrm{pH}, 7.6)$. The sections were then washed 3 times with PBS and mounted onto gelatin-coated slides. The slides were air-dried overnight at room temperature, and the coverslips were mounted using Permount (Fisher Scientific, Waltham, MA, USA).

\section{Histochemistry for NADPH-d}

To detect NOS activity in the PMC, vlPAG, and spinal cord (L5) regions, NADPH-d histochemistry was performed as previously described [20]. Briefly, free-floating sections were incubated at $37^{\circ} \mathrm{C}$ for 60 minutes in $100 \mathrm{mM}$ PBS $(\mathrm{pH}, 7.4)$ containing $0.3 \%$ Triton X-100, $0.1 \mathrm{mg} / \mathrm{mL}$ of nitroblue tetrazolium, and 0.1 $\mathrm{mg} / \mathrm{mL}$ of $\beta$-NADPH. The sections were then washed 3 times with PBS and mounted onto gelatin-coated slides. The slides were air-dried overnight at room temperature, and the coverslips were mounted using Permount (Fisher Scientific).

\section{Data Analysis}

To assess c-Fos, NGF and NADPH-d expressions in the neuronal afferent pathways of micturition (vlPAG, PMC, and spinal cord L5), cell counting was performed through a light micro- scope (BX-51; Olympus Corp., Tokyo, Japan). The area of the neuronal afferent pathways of micturition in each slice was measured using the Image-Pro Plus computer-assisted image analysis system (Media Cyberbetics Inc., Silver Spring, MD, USA) attached to the light microscope (BX-51; Olympus Corp.). The numbers of cells positive for c-Fos, NGF, and NADPH-d in the vlPAG and PMC were counted hemilaterally, and the numbers of cells positive for c-Fos, NGF, and NADPH$\mathrm{d}$ in the spinal cord $\mathrm{L} 5$ were counted on both sides of the dorsal horn. The results were expressed as the number in the selected region per section.

Statistical analysis was performed using IBM SPSS Statistics ver. 20.0 (IBM Co., Armonk, NY, USA). The data are expressed as the mean \pm standard error of the mean. For comparisons between groups, 1-way analysis of variance and the Duncan post hoc test were performed, and P-values $<0.05$ were considered to indicate statistically significant differences among the groups.

\section{RESULTS}

\section{Effects of Tamsulosin on Basal Contraction Pressure and Time According to Cystometry}

The basal contraction pressure and time according to cystometry are presented in Fig. 2. The basal contraction pressure and time in the SCI-induced group were significantly increased compared to the sham group $(\mathrm{P}<0.05)$. In contrast, tamsulosin treatments significantly decreased the basal contraction pressure and time, dose-dependently. In particular, treatment with $1 \mathrm{mg} / \mathrm{kg}$ of tamsulosin reduced the basal contraction pressure to a level similar to that of the sham group.

\section{Effects of Tamsulosin on c-Fos Positive Cells in the vIPAG, PMC, and Dorsal Horn of the Spinal Cord (L5)}

Photomicrographs of c-Fos-positive cells in the vlPAG, PMC, and dorsal horn of the spinal cord (L5) are presented in Fig. 3. These data showed that the expression of c-Fos in the vlPAG, PMC, and spinal dorsal horn (L5) of the SCI-induced group was conspicuously increased compared to the sham group $(\mathrm{P}<0.05)$. However, tamsulosin treatment significantly suppressed the expression of c-Fos in the vlPAG, PMC, and spinal dorsal horn (L5) in comparison to the SCI-induced group $(\mathrm{P}<0.05)$, and the inhibitory effect of tamsulosin on the expression of $\mathrm{c}$-Fos in the spinal cord was dose-dependent. 

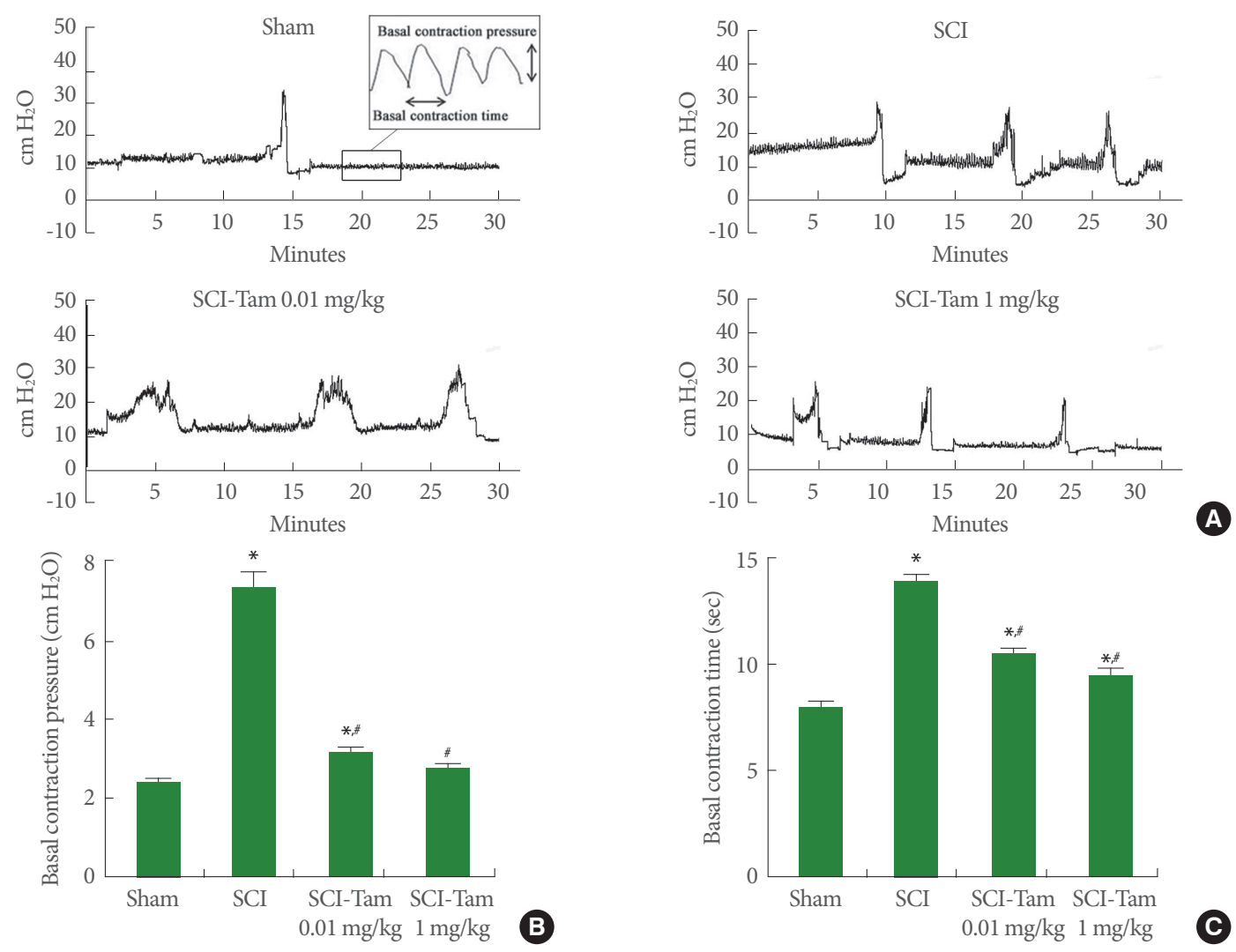

Fig. 2. Effects of tamsulosin on basal contraction pressure and time in cystometry. (A) Representative graph of cystometry in each group. (B) Effects of tamsulosin on the basal contraction pressure. (C) Effects of tamsulosin on the basal contraction time. Sham, the sham group; SCI, the SCI-induced group; SCI-Tam $0.01 \mathrm{mg} / \mathrm{kg}$, the SCI-induced and $0.01 \mathrm{mg} / \mathrm{kg}$ tamsulosin-treated group; and SCITam $1 \mathrm{mg} / \mathrm{kg}$, the SCI-induced and $1 \mathrm{mg} / \mathrm{kg}$ tamsulosin-treated group. The results are presented as the mean \pm standard error of the mean. SCI, spinal cord injury. ${ }^{*} \mathrm{P}<0.05$ compared to the sham group. ${ }^{*} \mathrm{P}<0.05$ compared to the SCI-induced group.

\section{Effects of Tamsulosin on NGF-Positive Cells in the vIPAG, PMC, and Dorsal Horn of the Spinal Cord (L5)}

Photomicrographs of NGF-positive cells in the vlPAG, PMC, and spinal dorsal horn (L5) are presented in Fig. 4. Similar to the expression of c-Fos, the expression of NGF in the vlPAG, PMC, and spinal dorsal horn (L5) of the SCI-induced group was remarkably increased, compared to the sham group $(\mathrm{P}<0.05)$. Moerover, tamsulosin treatment significantly suppressed the expression of NGF in the vlPAG, PMC, and spinal dorsal horn (L5) compared to the SCI-induced group $(\mathrm{P}<0.05)$. This suppression of NGF expression in the vlPAG, PMC, and dorsal horn of the spinal cord by tamsulosin was proportional to the doses of these agents.

\section{Effects of Tamsulosin on NADPH-d Positive Cells in the vIPAG, PMC, and Dorsal Horn of the Spinal Cord (L5)}

Photomicrographs of NADPH-d-positive cells in the vlPAG,
PMC, and dorsal horn of the spinal cord (L5) are presented in Fig. 5. While the expression of NADPH-d in the vlPAG, PMC, and spinal dorsal horn (L5) of the SCI-induced group remarkably increased, tamsulosin treatment significantly suppressed the expression of NADPH-d in the vlPAG, PMC, and spinal dorsal horn compared to the SCI-induced group $(\mathrm{P}<0.05)$. Tamsulosin treatment reduced the number of NADPH-d-positive cells in the vlPAG, PMC, and spinal dorsal horn to the level of the sham group.

\section{DISCUSSION}

Among the many experimental SCI models that have been developed, weight drop methods and the clip-damage model after laminectomy resulted in low survival rates due to various side effects after surgery. Therefore, in this study, SCI was induced by the method of Cho et al. [19]. The advantage of this model is 

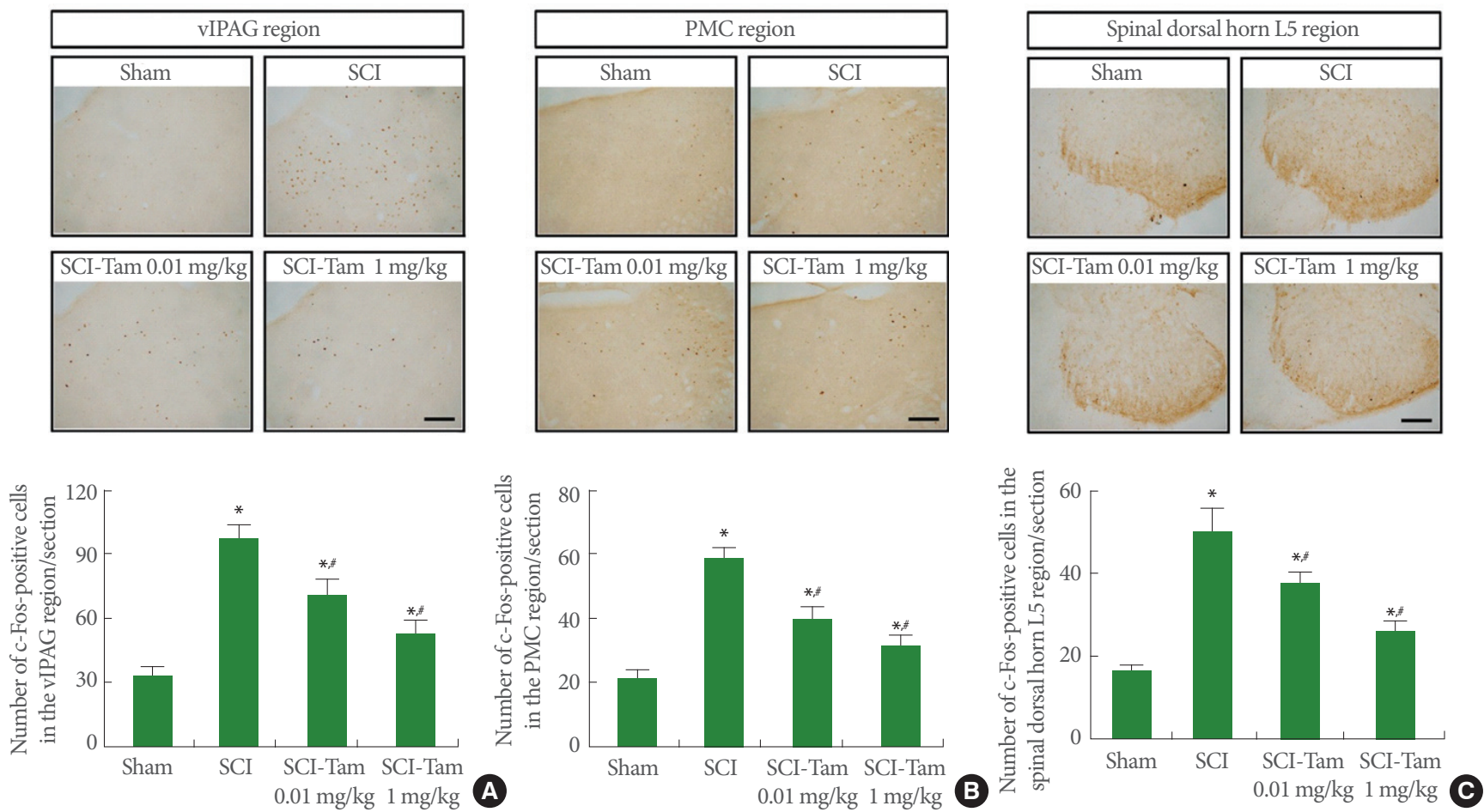

Fig. 3. Effects of tamsulosin on the expression of c-Fos in the vlPAG, PMC, and dorsal horn of the spinal cord (L5). (A) vlPAG region, (B) PMC region, (C) Dorsal horn of the spinal cord. Upper: Representative photomicrographs of c-Fos-positive cells in each group. The scale bar represents $200 \mu \mathrm{m}$. Lower: Effect of tamsulosin on the expression of c-Fos in the vlPAG, PMC, and spinal dorsal horn of each group. Sham, the sham group; SCI, the SCI-induced group; SCI-Tam $0.01 \mathrm{mg} / \mathrm{kg}$, the SCI-induced and $0.01 \mathrm{mg} / \mathrm{kg}$ tamsulosintreated group; and SCI-Tam $1 \mathrm{mg} / \mathrm{kg}$, the SCI-induced and $1 \mathrm{mg} / \mathrm{kg}$ tamsulosin-treated group. The results are presented as the mean \pm standard error of the mean. vlPAG, ventrolateral periaqueductal gray matter; PMC, pontine micturition center; SCI, spinal cord injury. ${ }^{\star} \mathrm{P}<0.05$ compared to the sham group. ${ }^{*} \mathrm{P}<0.05$ compared to the $\mathrm{SCI}$-induced group.

that it has a high survival rate of animals because of the rapid operation and because the affected part is minimized.

SCI impairs the neuronal pathways running through the spinal cord, and animal models of SCI induced by chemical lesions, induction of ischemia, and complete or incomplete transection are used to study pathologies related to SCI, including bladder dysfunction $[11,12]$. SCI has been reported to induce substantial anatomical reorganization of spinal circuits, to affect the imbalance between excitatory and inhibitory inputs, and to impair certain cellular functions [21]. Consequently, SCI significantly impairs micturition and results in areflexic detrusor underactivity and neurogenic detrusor overactivity, depending the level of the lesion [12]. de Groat and Yoshimura [13] reported that SCI at the cervical or thoracic levels disrupted voluntary control of voiding and the normal reflex pathways that coordinate bladder and sphincter functions. Miyazato et al. [14] also reported that SCI induced by transection of T9-10 significantly increased non-voiding bladder contractions and decreased bladder capacity. Similarly, the present study found that SCI induced by incomplete transection of T11 significantly increased the basal contraction pressure and time. Such increases in the basal contraction pressure and time have been found in cyclophosphamide-induced $\mathrm{OAB}$ and the spontaneous hypertensive rat animal model of detrusor overactivity and $\mathrm{OAB}[20,22]$.

Previous studies suggested that these afferent signals and their central processing play an important role in normal LUT function and in the pathogenesis of functional LUT diseases, such as $\mathrm{OAB}$ and neurogenic bladder [11-13,17]. Following SCI, the bladder is initially a reflexic but then becomes hyperreflexic, due to the emergence of a spinal micturition reflex pathway [13]. Sensory afferent fibers run in the 3 sets of peripheral nerves - pelvic, hypogastric, and pudendal - and transmit information to the CNS about bladder filling. Most of the bladder sensory fibers run in the pelvic nerve, and their cell bodies are 

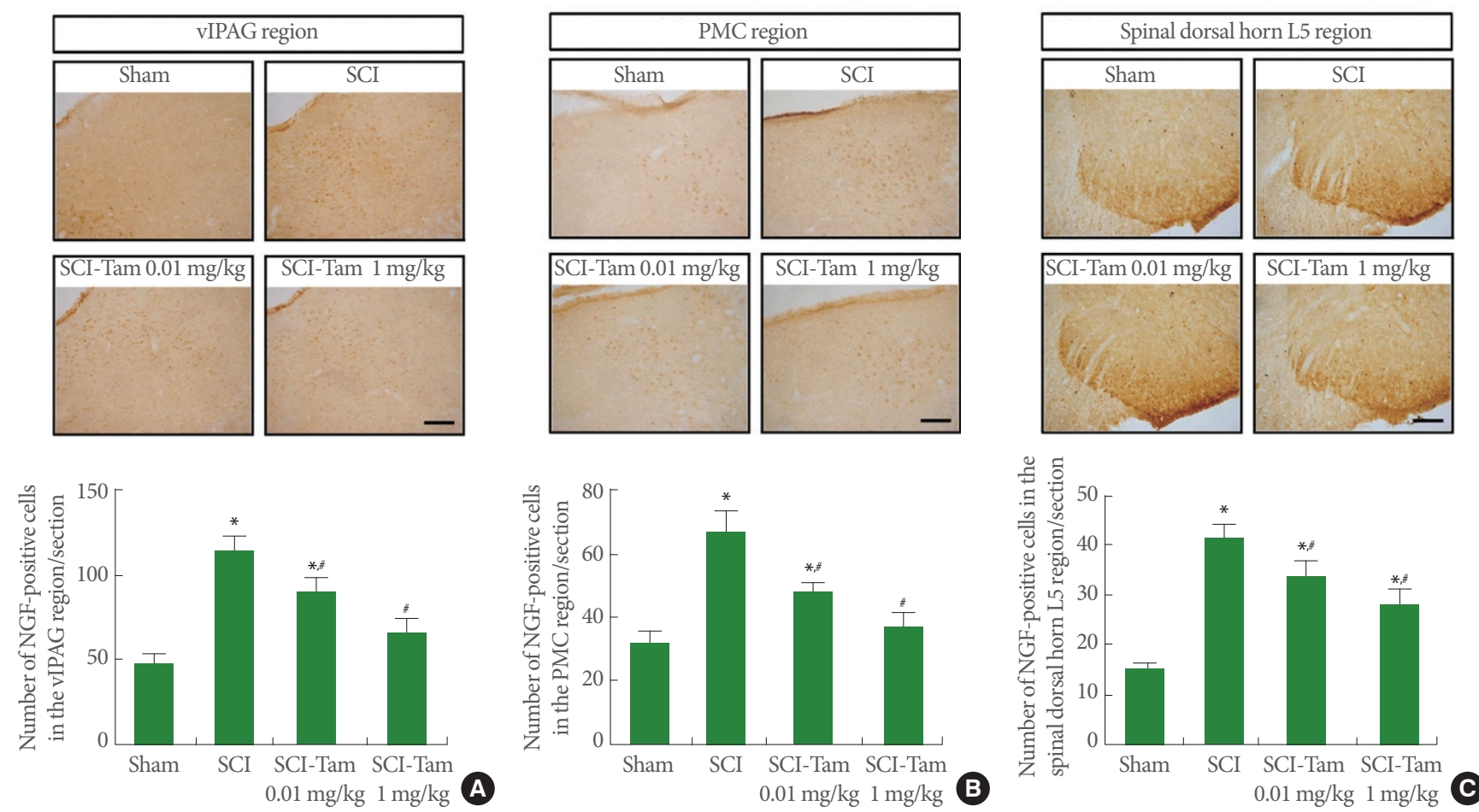

Fig. 4. Effects of tamsulosin on the expression of NGF in the vlPAG, PMC, and dorsal horn of the spinal cord (L5). (A) vlPAG region, (B) PMC region, (C) Dorsal horn of the spinal cord. Upper: Representative photomicrographs of NGF-positive cells in each group. The scale bar represents $200 \mu \mathrm{m}$. Lower: Effect of tamsulosin on the expression of NGF in the vlPAG, PMC, and spinal dorsal horn of each group. Sham, the sham group; SCI, the SCI-induced group; SCI-Tam $0.01 \mathrm{mg} / \mathrm{kg}$, the SCI-induced and $0.01 \mathrm{mg} / \mathrm{kg}$ tamsulosintreated group; and SCI-Tam $1 \mathrm{mg} / \mathrm{kg}$, the SCI-induced and $1 \mathrm{mg} / \mathrm{kg}$ tamsulosin-treated group. The results are presented as the mean \pm standard error of the mean. NGF, nerve growth factor; vlPAG, ventrolateral periaqueductal gray matter; PMC, pontine micturition center; SCI, spinal cord injury. ${ }^{\star} \mathrm{P}<0.05$ compared to the sham group. ${ }^{*} \mathrm{P}<0.05$ compared to the SCI-induced group.

located in the sacral dorsal root ganglia in humans and in L5$S 1$ in rats [12]. These sacral afferent fibers send their projections to the PAG, via the supraspinal pathway [4]. The bladder information is first processed within the PAG, and then it is relayed to the PMC. The PMC, as the supraspinal switching center, sends excitatory motor input to the sacral spinal cord, resulting in detrusor contraction and relaxation of the urethra $[3,23]$. More specifically, the bladder, via the lumbosacral spinal cord, sends information about bladder filling to the PAG, and then PMC-projecting cells in the PAG become activated and initiate micturition.

Afferent fibers from the bladder have been reported to express tropomyosin-related kinase $\mathrm{A}$, a high-affinity receptor for NGF [24]. NGF has been reported to modulate neuronal function associated with micturition and to be involved in the development of neurogenic bladder overactivity at the spinal level [25]. Yoshimura et al. [26] reported that increased NGF levels in bladder afferent pathways and NGF-induced reduction in Atype $\mathrm{K}+$ current density could contribute to the hypertrophy and hyperexcitability of bladder afferent neurons, as well as to the emergence of bladder overactivity. Our results also showed that the expression of NGF in the spinal dorsal horn of the SCIinduced group was conspicuously increased in comparison with the sham group, and, similarly to NGF expression in the lumbosacral spinal cord, NGF expression was upregulated in the vIPAG and PMC. The levels of NGF are strongly enhanced in SCI animals, and alterations in the synthesis and release of neurotrophic factors in the peripheral and central nervous system could contribute to the emergence of the spinal micturition reflex [27].

de Groat and Yoshimura [10] suggested that the expression of c-Fos in the spinal cord identifies that the spinal neurons are involved in processing afferent input from the LUT. Such an increase of neuronal activities in the lumbosacral spinal cord may 

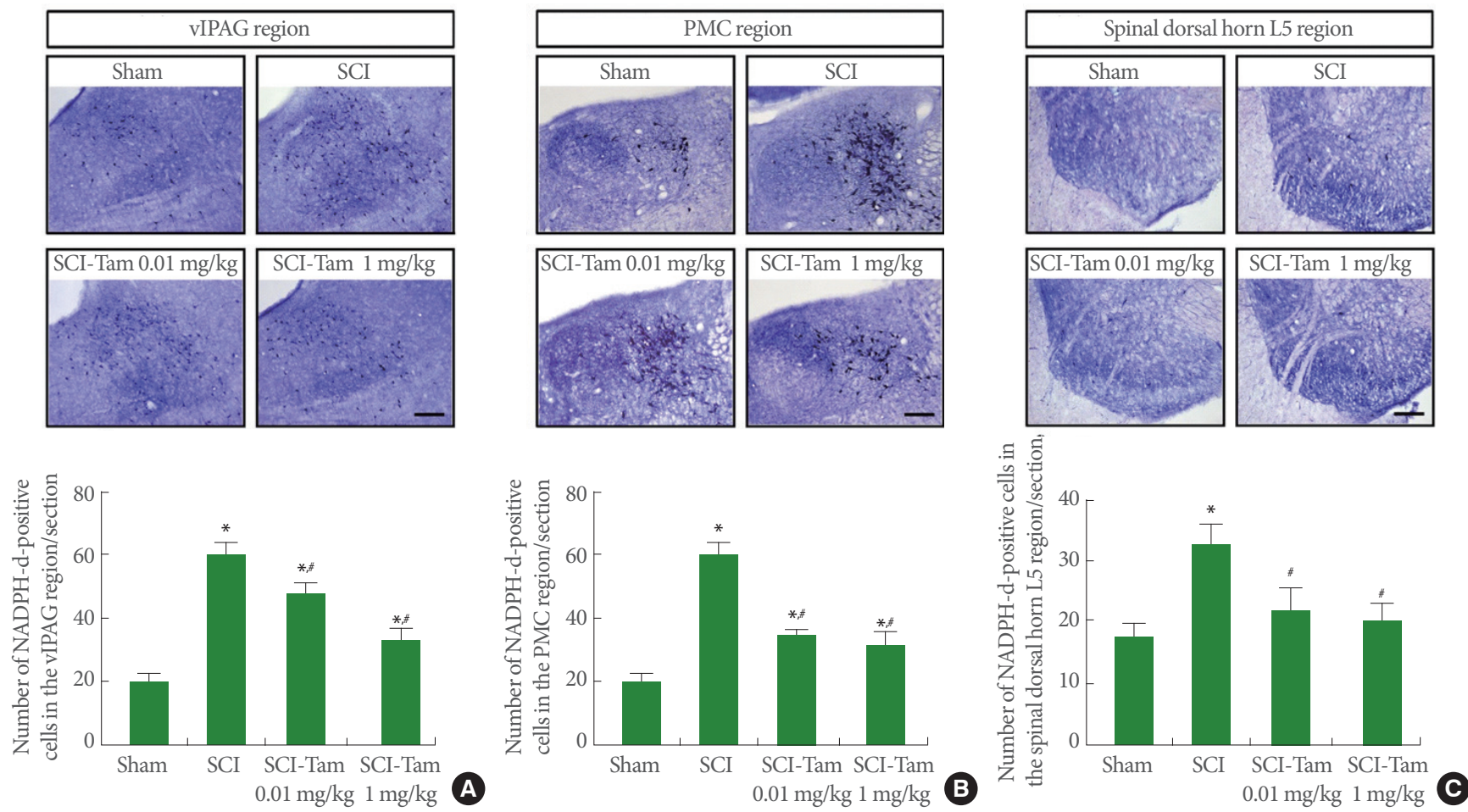

Fig. 5. Effects of tamsulosin on the expression of NADPH-d in the vlPAG, PMC, and dorsal horn of the spinal cord (L5). (A) vlPAG region, (B) PMC region, (C) Dorsal horn of the spinal cord. Upper: Representative photomicrographs of NADPH-d-positive cells in each group. The scale bar represents $200 \mu \mathrm{m}$. Lower: Effect of tamsulosin on the expression of NADPH-d in the vlPAG, PMC, and spinal dorsal horn of each group. Sham, the sham group; SCI, the SCI-induced group; SCI-Tam $0.01 \mathrm{mg} / \mathrm{kg}$, the SCI-induced and 0.01 $\mathrm{mg} / \mathrm{kg}$ tamsulosin-treated group; and SCI-Tam $1 \mathrm{mg} / \mathrm{kg}$, the SCI-induced and $1 \mathrm{mg} / \mathrm{kg}$ tamsulosin-treated group. The results are presented as the mean \pm standard error of the mean. NADPH-d, nicotinamide adenine dinucleotide phosphate-diaphorase; vlPAG, ventrolateral periaqueductal gray matter; $\mathrm{PMC}$, pontine micturition center; $\mathrm{SCI}$, spinal cord injury. ${ }^{\star} \mathrm{P}<0.05$ compared to the sham group. ${ }^{*} \mathrm{P}<0.05$ compared to the $\mathrm{SCI}$-induced group.

stimulate the supraspinal pathways regulating micturition. In fact, many studies have reported that stimulation of the bladder caused the expression of c-Fos in the PAG and PMC [3,5]. Microinjections of excitatory neurotransmitters, such as glutamate, acetylcholine, or $\gamma$-aminobutyric acid antagonist, into the PMC induce bladder contraction, decreases the threshold bladder volume causing the micturition reflex, or increase the amplitude of bladder contraction [28]. Based on these studies, it can be suggested that stimulation or enhanced neuronal activity in the PMC and PAG might be involved in the symptoms of OAB. In the present results, the expression of $\mathrm{c}$-Fos in the spinal dorsal horn, vlPAG, and PMC in the SCI-induced group was significantly upregulated compared to the sham group, and the basal contraction pressure and time were greater in the SCI-induced group than in the sham group.

SCI can induce the upregulated expression of NOS, and of particular note, the upregulation of nNOS may facilitate the emergence of the spinal micturition reflex following SCI [9]. Kakizaki and de Groat [29] suggested that NO at the spinal level participates in the facilitation of the micturition reflex by nociceptive bladder afferent neurons activated by noxious chemical irritation of the bladder. Lagos and Ballejo [30] also reported that the increased production of NO in spinal L6-S2 segments seems to be necessary for the initiation of micturition hyperreflexia based on the identification of increased $\mathrm{Ca}^{2+}$-dependent NOS activity in the micturition hyperreflexia observed in the initial hours of cyclophosphamide-induced cystitis. Neurons containing NO have been identified histochemically by the presence of NADPH-d [31], and such NADPH-d activity has also been identified in neurons in the spinal cord [29]. The present study also showed that the expression of NADPH-d in the spinal dorsal horn was conspicuously upregulated following SCI, suggesting an increased production of NO. In addition, upregulation of NADPH-d occurred in the vIPAG and PMC, 
the supraspinal afferent pathways.

As shown in this study, SCI upregulated neural activities and the production of NO in the sacral afferent pathway (L5) and the supraspinal pathways, the vlPAG and PMC, resulting in OAB. However, tamsulosin treatment significantly suppressed the expression of c-Fos and NGF in the dorsal horn of the spinal cord (L5), the vlPAG, and the PMC. Moreover, tamsulosin treatment remarkably reduced the expression of NADPH-d in the afferent pathways of micturition. Consequently, tamsulosin significantly attenuated the symptoms of OAB.

Activation of the al-adrenoceptor in the bladder causes OAB symptoms [32]. Thus, tamsulosin, an a1-adrenoceptor antagonist, has been reported to have beneficial effects on the micturition function [20,22]. Yokoyama et al. [17] reported that tamsulosin had an inhibitory effect on the C-fiber urethral afferent nerves, resulting in improved bladder storage. Most sensory afferent nerves innervating the LUT, especially the bladder, are small myelinated (A $\delta$ fibers) or unmyelinated (C fibers) axons. In particular, $\mathrm{C}$ fibers can be identified by their neuropeptide content and expression of transient receptor potential vanilloid 1 [33]. In chronic SCI rats, C-fiber afferent neurons mediate bladder hyperreflexia and automatic micturition, leading to detrusor-sphincter dyssynergia, which refers to simultaneous contractions of the external urethral sphincter and bladder during the micturition reflex $[13,34]$.

Seki et al. [34] reported that increased levels of NGF in the spinal cord are involved in the emergence of detrusor-sphincter dyssynergia which is partially mediated by C-fiber bladder afferent neurons after SCI. NGF has been reported to induce hyperexcitability in C-fiber bladder afferent pathways and bladder hyperactivity in various pathological conditions, including SCI $[27,34]$. Yoshimura et al. [26] also reported that increased NGF levels in bladder afferent pathways and NGF-induced reduction in A-type $\mathrm{K}^{+}$current density could contribute to the hyperexcitability of bladder afferent neurons and bladder overactivity. However, neutralization of NGF in the spinal cord suppressed C-fiber mediated bladder hyperreflexia in SCI-induced rats [34]. Thus, suppression of NGF levels in afferent pathways could be useful for treating detrusor-sphincter dyssynergia or voiding dysfunction following SCI $[10,34]$. In the present study, tamsulosin treatment also significantly suppressed the expression of NGF in the dorsal horn of the spinal cord (L5) in SCIinduced rats, and this inhibitory effect of tamsulosin on NGF expression also occurred in the supraspinal pathways, the vlPAG, and the PMC. In addition, tamsulosin suppressed the ex- pressions of c-Fos in the spinal cord (L5), the vlPAG and the PMC. Suppressing the expression of c-Fos in afferent pathways regulating micturition contributes to the attenuation of $\mathrm{OAB}$ symptoms, and this inhibitory effect of tamsulosin has been found in other studies $[20,22]$.

Finally, tamsulosin suppressed the expression of NADPH-d in the dorsal horn of the spinal cord (L5), the vlPAG, and the PMC to a remarkable extent, suggesting that NO production was suppressed in these regions. C-fiber afferent neurons are sensitive to NO, and the increased production of $\mathrm{NO}$ may be necessary for the initiation of micturition hyperreflexia [30]. Moreover, the production of NO seems to be associated with increased spinal Fos expression [35]. Lagos and Ballejo [30] found that the nonselective NOS inhibitor NG-nitro-L-arginine-methyl ester diminished the hyperreflexic state when administered systemically, and Zhang et al. [9] also reported that an nNOS inhibitor suppressed SCI-induced urinary incontinence by increasing bladder capacity.

The results of the present study demonstrate that SCI increased nonvoiding bladder contractions and reduced bladder capacity by increasing the expression of NGF and c-Fos and NO production in the afferent pathways of micturition, which suggests that the bladder afferent neurons became hyperexcitable and that $\mathrm{OAB}$ was induced. However, tamsulosin treatment showed beneficial effects on alleviating the symptoms associated with bladder hyperactivity by suppressing hyperexcitability in the afferent pathways of micturition (lumbosacral spinal cord, the vlPAG, and the PMC). Therefore, tamsulosin, an a1-adrenoceptor antagonist, may be able to attenuate bladder dysfunction following SCI. However, this study did not investigate the other regions involved in micturition, such as the anterior cingulate gyrus, medial preoptic nucleus, and frontal cortex. Thus, further studies are needed to elucidate the exact mechanism and role of tamsulosin on the afferent pathways of micturition.

\section{REFERENCES}

1. Rickey LM, Sarkey S, DonCarlos LL. Estrogen-sensitive projections from the medial preoptic area to the dorsal pontine tegmentum, including Barrington's nucleus, in the rat. Neurourol Urodyn 2008; 27:440-5.

2. Marson L. Identification of central nervous system neurons that innervate the bladder body, bladder base, or external urethral sphincter of female rats: a transneuronal tracing study using pseudorabies 
virus. J Comp Neurol 1997;389:584-602.

3. Kavia RB, Dasgupta R, Fowler CJ. Functional imaging and the central control of the bladder. J Comp Neurol 2005;493:27-32.

4. Blok BF. Brain control of the lower urinary tract. Scand J Urol Nephrol Suppl 2002;(210):11-5.

5. Saban MR, Nguyen NB, Hammond TG, Saban R. Gene expression profiling of mouse bladder inflammatory responses to LPS, substance P, and antigen-stimulation. Am J Pathol 2002;160:2095-110.

6. Hiroi N, Brown JR, Haile CN, Ye H, Greenberg ME, Nestler EJ. FosB mutant mice: loss of chronic cocaine induction of Fos-related proteins and heightened sensitivity to cocaine's psychomotor and rewarding effects. Proc Natl Acad Sci USA 1997;94:10397-402.

7. Steers WD, Tuttle JB. Mechanisms of disease: the role of nerve growth factor in the pathophysiology of bladder disorders. Nat Clin Pract Urol 2006;3:101-10.

8. Ho MH, Bhatia NN, Khorram O. Physiologic role of nitric oxide and nitric oxide synthase in female lower urinary tract. Curr Opin Obstet Gynecol 2004;16:423-9.

9. Zhang F, Liao L, Ju Y, Song A, Liu Y. Neurochemical plasticity of nitric oxide synthase isoforms in neurogenic detrusor overactivity after spinal cord injury. Neurochem Res 2011;36:1903-9.

10. de Groat WC, Yoshimura N. Changes in afferent activity after spinal cord injury. Neurourol Urodyn 2010;29:63-76.

11. de Groat WC, Yoshimura N. Afferent nerve regulation of bladder function in health and disease. Handb Exp Pharmacol 2009;(194): 91-138.

12. Cruz CD, Cruz F. Spinal cord injury and bladder dysfunction: new ideas about an old problem. ScientificWorldJournal 2011;11:21434.

13. de Groat WC, Yoshimura N. Mechanisms underlying the recovery of lower urinary tract function following spinal cord injury. Prog Brain Res 2006;152:59-84.

14. Miyazato M, Oshiro T, Chancellor MB, de Groat WC, Yoshimura N, Saito S. An alpha1-adrenoceptor blocker terazosin improves urine storage function in the spinal cord in spinal cord injured rats. Life Sci 2013;92:125-30.

15. Yazaki J, Aikawa K, Shishido K, Yanagida T, Nomiya M, Ishibashi $\mathrm{K}$, et al. Alpha1-adrenoceptor antagonists improve bladder storage function through reduction of afferent activity in rats with bladder outlet obstruction. Neurourol Urodyn 2011;30:461-7.

16. Roehrborn CG, Siami P, Barkin J, Damião R, Major-Walker K, Morrill B, et al. The effects of dutasteride, tamsulosin and combination therapy on lower urinary tract symptoms in men with benign prostatic hyperplasia and prostatic enlargement: 2-year results from the CombAT study. J Urol 2008;179:616-21.
17. Yokoyama O, Yusup A, Oyama N, Aoki Y, Miwa Y, Akino H. Improvement in bladder storage function by tamsulosin depends on suppression of C-fiber urethral afferent activity in rats. J Urol 2007; 177:771-5.

18. Abrams P, Amarenco G, Bakke A, Buczyński A, Castro-Diaz D, Harrison S, et al. Tamsulosin: efficacy and safety in patients with neurogenic lower urinary tract dysfunction due to suprasacral spinal cord injury. J Urol 2003;170(4 Pt 1):1242-51.

19. Cho YS, Ko IG, Kim SE, Lee SM, Shin MS, Kim CJ, et al. Oral mucosa stem cells alleviates spinal cord injury-induced neurogenic bladder symptoms in rats. J Biomed Sci 2014;21:43.

20. Kim SE, Shin MS, Kim CJ, Park JH, Chung KJ, Jung H, et al. Effects of tamsulosin on urinary bladder function and neuronal activity in the voiding centers of rats with cyclophosphamide-induced overactive bladder. Int Neurourol J 2012;16:13-22.

21. Yezierski RP. Spinal cord injury pain: spinal and supraspinal mechanisms. J Rehabil Res Dev 2009;46:95-107.

22. Kim SE, Ko IG, Hwang L, Choi IY, Shin MS, Kim CJ, et al. An animal study to compare the degree of the suppressive effects on the afferent pathways of micturition between tamsulosin and sildenafil. J Biomed Sci 2013;20:81.

23. Fowler CJ, Griffiths DJ. A decade of functional brain imaging applied to bladder control. Neurourol Urodyn 2010;29:49-55.

24. Qiao LY, Vizzard MA. Spinal cord injury-induced expression of TrkA, TrkB, phosphorylated CREB, and c-Jun in rat lumbosacral dorsal root ganglia. J Comp Neurol 2005;482:142-54.

25. Ochodnický P, Cruz CD, Yoshimura N, Michel MC. Nerve growth factor in bladder dysfunction: contributing factor, biomarker, and therapeutic target. Neurourol Urodyn 2011;30:1227-41.

26. Yoshimura N, Bennett NE, Hayashi Y, Ogawa T, Nishizawa O, Chancellor MB, et al. Bladder overactivity and hyperexcitability of bladder afferent neurons after intrathecal delivery of nerve growth factor in rats. J Neurosci 2006;26:10847-55.

27. Vizzard MA. Neurochemical plasticity and the role of neurotrophic factors in bladder reflex pathways after spinal cord injury. Prog Brain Res 2006;152:97-115.

28. Sugaya K, Nishijima S, Miyazato M, Ogawa Y. Central nervous control of micturition and urine storage. J Smooth Muscle Res 2005;41:117-32.

29. Kakizaki H, de Groat WC. Role of spinal nitric oxide in the facilitation of the micturition reflex by bladder irritation. J Urol 1996; 155:355-60.

30. Lagos P, Ballejo G. Role of spinal nitric oxide synthase-dependent processes in the initiation of the micturition hyperreflexia associated with cyclophosphamide-induced cystitis. Neuroscience 2004; 
125:663-70.

31. Vincent SR, Kimura H. Histochemical mapping of nitric oxide synthase in the rat brain. Neuroscience 1992;46:755-84.

32. Andersson KE. Overactive bladder-pharmacological aspects. Scand J Urol Nephrol Suppl 2002;(210):72-81.

33. Avelino A, Cruz C, Nagy I, Cruz F. Vanilloid receptor 1 expression in the rat urinary tract. Neuroscience 2002;109:787-98.
34. Seki S, Sasaki K, Igawa Y, Nishizawa O, Chancellor MB, de Groat WC, et al. Suppression of detrusor-sphincter dyssynergia by immunoneutralization of nerve growth factor in lumbosacral spinal cord in spinal cord injured rats. J Urol 2004;171:478-82.

35. Callsen-Cencic P, Mense S. Increased spinal expression of c-Fos following stimulation of the lower urinary tract in chronic spinal cord-injured rats. Histochem Cell Biol 1999;112:63-72. 